\title{
Medienkompetenz oder Medienbildung? \\ Zur Frage nach dem Zielwert medienpädagogischer Praxis
}

Marius Thomann

\begin{abstract}
Zusammenfassung
In einer Serie von vier Artikeln (Schorb 2009; Spanhel 2010; Tulodziecki 2010; Fromme u. Jörissen 2010) wurde 2009 und 2010 in der Zeitschrift merz: Medien + Erziehung die Frage nach dem Leitbegriff der Medienpädagogik diskutiert. In den vorliegenden Ausführungen wird im Bezugsrahmen dieser Debatte die Frage diskutiert, ob Medienkompetenz oder Medienbildung der Zielwert medienpädagogischer Praxis sein sollte. Dabei wird in einer argumentationstheoretischen Analyse zunächst jeweils ein deskriptives und ein normatives Argument gegen Medienkompetenz rekonstruiert und zurückgewiesen. Als Grundlage dieser Argumente zeigt sich hierbei eine begriffliche Verwechslung zwischen den theoretischen Modellen zur Medienkompetenz und der Verwendung dieses Konzepts im pädagogisch verkürzten Sinne einzelner Unterkompetenzen. Anschliessend werden drei Adäquatheitskriterien für Zielwerte medienpädagogischer Praxis vorgeschlagen, die dann als Grundlage für die Bewertung beider Alternativen im begrifflichen Bezugsrahmen der genannten Debatte dienen. Im Ergebnis empfiehlt sich Medienkompetenz als dieser Zielwert, während das Konzept der Medienbildung begrifflicher Schärfung und einer Ausarbeitung seiner Schnittstelle zur medienpädagogischen Praxis bedarf.
\end{abstract}

\section{Media Competence or Media Literacy?} On the question regarding the target value of applied media pedagogy

\begin{abstract}
In a series of four papers (Schorb 2009; Spanhel 2010; Tulodziecki 2010; Fromme and Jörissen 2010) the question regarding the main term in media pedagogy was discussed in the journal merz: Medien + Erziehung in 2009 and 2010. With reference to that debate, the present paper discusses the question whether media competence (German Medienkompetenz) or media literacy (German Medienbildung) should be the target value of applied media pedagogy. In doing so, a descriptive and a normative argument against media competence are reconstructed from the perspective of argumentation theory and both of them are refuted. The basis of these arguments turns out to be a conceptual confusion of the
\end{abstract}


theoretical models of media competence with the use of this concept in the sense of sub-competences, the latter being pedagogically inadequate. After that, three criteria of adequacy are suggested for target values of applied media pedagogy. Those criteria serve as a measure to evaluate both alternatives in the conceptual framework of the debate mentioned above. As a result, media competence commends itself as that target value. Media literacy, on the other hand, is in need of a more pronounced conception and an elaboration of its interface to applied media pedagogy.

\section{Warum überhaupt Medienpädagogik?}

Eine Beobachtung vorweg: Sozialisation ist heutzutage immer häufiger mediale Sozialisation und, damit verbunden bzw. durch die Möglichkeiten der sogenannten Neuen Medien verstärkt, in immer grösseren Teilen ein Prozess, der selbstbestimmt durchlaufen wird (vgl. Fromme 2009, 931 ff.). Diese Diagnose soll hier ebenso wenig vertieft werden wie die systematische Grundannahme der folgenden Ausführungen: Zu den Hauptaufgaben der Pädagogik im Allgemeinen gehört es, die Entwicklung der individuellen Persönlichkeit und somit auch den Prozess der Sozialisation transparent zu machen und zu begleiten. Auf der Basis dieser beiden Annahmen ergibt sich die Wichtigkeit einer theoretisch fundierten und praktisch anschlussfähigen Medienpädagogik. Pädagogisches Handeln muss den Anschluss an die Lebenswelt des Individuums suchen, damit es intrinsische Motivation wecken kann und nicht als externe Verordnung ohne Bezug zum jeweils eigenen Kontext empfunden wird. Diese Anbindung an den lebensweltlichen Kontext erfordert einerseits die theoretische Aufarbeitung der pädagogischen Bedeutung medialer Räume sowie eine Analyse der pädagogisch relevanten Eigenschaften von Medien und andererseits eine Verständigung darüber, was in der pädagogischen Praxis letztlich geleistet werden soll, was geleistet werden kann und welche Massnahmen dafür geeignet sind. Und damit ist das Feld der Medienpädagogik eröffnet.

Im Folgenden geht es um die Frage nach dem Zielwert der Medienpädagogik, genauer der medienpädagogischen Praxis. Eine Diskussion über den Leitbegriff medienpädagogischer Theoriebildung kann hier nicht stattfinden. Als mögliche Alternativen werden Medienkompetenz und Medienbildung im begrifflichen Bezugsrahmen der jüngeren Debatte (vgl. z. B. Hugger 2006; Schorb 2009; Spanhel 2010; Tulodziecki 2010; Fromme u. Jörissen 2010) diskutiert. Zunächst werden in einer argumentationstheoretischen Analyse zwei Argumente rekonstruiert, deren Ziel es ist, die Medienkompetenz als Zielwert der Medienpädagogik zu disqualifizieren. Jeweils im Anschluss an die Rekonstruktionen wird es darum gehen, diesen Argumenten zu begegnen, um so die Möglichkeit offenzuhalten, den Zielwert als Medienkompetenz zu bestimmen. Abschliessend wird, ebenfalls unter Bezugnahme auf den konzeptuellen Rahmen der genannten Debatte, der Versuch einer po- 
sitiven Argumentation für diese Bestimmung unternommen. Systematisch versteht sich dieser Beitrag also als Analyse eines konkreten innertheoretischen Diskurses, die um eine durch Kriterien geleitete Bewertung angereichert ist.

\section{Zwei Argumente gegen Medienkompetenz als Zielwert}

Bezüglich der Trennung zwischen Medienkompetenz und Medienbildung soll der Fokus hier auf zwei Aspekten liegen, und zwar erstens auf der Unterscheidung zwischen Lern- und Bildungsprozessen und zweitens auf den unterschiedlichen motivationalen Eigenschaften solcher Prozesse. Die Differenz zwischen den zur Diskussion stehenden Konzepten wird häufig vor dem Hintergrund von Verschiedenheiten in diesen beiden Dimensionen gesehen. Gleichzeitig erlauben diese Abgrenzungen, zwei Argumente verschiedenen Typs zu formulieren, nämlich ein deskriptives und ein normatives Argument, so dass hier verschiedene Stossrichtungen der Kritik berücksichtigt werden können.

\section{Das deskriptive Argument: Lernprozesse vs. Bildungsprozesse}

Lernprozesse zielen auf den Erwerb sogenannten Verfügungswissens und das jeweils in Bezug auf einen bestimmten abgegrenzten Gegenstandsbereich (vgl. Fromme 2009, 934). Mit dem Begriff des Verfügungswissens ist hier einerseits propositional verfasstes Wissen-dass gemeint, also z. B. Wissen um die Tatsache, dass Julius Cäsar am 15. März im Jahre 44 v. Chr. ermordet wurde, andererseits aber auch Wissen-wie, d. h. Fähigkeiten wie z. B. problemorientiertes Quellenstudium in der Geschichtswissenschaft. Durch die für Lernprozesse charakteristische Bezogenheit auf einen Gegenstandsbereich sind die Inhalte, die sich das Individuum in ihnen aneignet, immer auf der Objektebene angesiedelt. Im Gegensatz dazu ist in Bildungsprozessen immer auch der Gegenstandsbereich selbst thematisch, nicht bloss seine Inhalte (vgl. ebd.). Ziel ist dabei nicht ein inhaltlich orientiertes Verfügungswissen, sondern höherstufiges Orientierungswissen, das auf die Position des Individuums in und sein Verhältnis zu der Welt bezogen und somit wesentlich auf der Metaebene zu verorten ist.

Medienkompetenz, so behauptet Fromme, meint in erster Linie «die aktive Erschliessung und Aneignung der für den Einzelnen relevanten Bereiche in der heutigen Medienwelt» (ebd.). Sie ist damit parallel zu den oben umrissenen Lernprozessen zu verstehen bzw. als deren Ziel. In diesem Verständnis entspricht Medienkompetenz dem Bildungsbegriff des Diskurses, den Jörissen als «praxistheoretisch-pädagogisch» $(2011,212)$ bezeichnet und in dem Bildung «im Sinne eines Ergebnisses oder Zieles von (in [sic!] weitesten Sinne) pädagogischen Handlungen» (ebd., 215, Hervorh. im Original) gefasst wird. Das Konzept der Medienbildung übersteigt das der Medienkompetenz, indem es über die Inhalte auf der Objektebene hinaus auch die Wirkung sowohl der Inhalte als auch ihrer medialen Vermittlung auf die 
Struktur von Welt- und Selbstverhältnis des Individuums erfasst. Indem Bildungsprozesse wie oben beschrieben den Gegenstandsbereich selbst thematisieren, sind sie die «Bedingung der Möglichkeit für eine kritische Reflexion des in einem Weltbereich geltenden Wissens und Voraussetzung für den Aufbau von Orientierungswissen» (Fromme 2009, 934). Medienbildungsprozesse sind also Prozesse, in denen sich das Individuum selbstbestimmt und bewusst in ein Verhältnis zu seiner medialen Lebenswelt setzt (vgl. Hugger 2006, 31). Dabei bestimmt es seine eigene Position und Rolle, seine Verantwortlichkeiten und Möglichkeiten in Bezug sowohl auf die medial vermittelten Inhalte als auch auf die mediale Vermittlung von Inhalten. Als wesentliches Merkmal von Medienbildung wird ihr prozesshafter Charakter genannt (vgl. z. B. Spanhel 2010, 50; Jörissen 2011, 222), d. h., Medienbildung zielt gerade nicht auf einen Zustand, sondern ermöglicht eine Offenheit, in der das Welt- und Selbstverhältnis immer wieder neu definiert werden kann.

Auf der Basis dieser Charakterisierungen von Medienkompetenz und Medienbildung lässt sich nun das erste Argument gegen Medienkompetenz als Zielwert medienpädagogischer Praxis rekonstruieren: Medienkompetenz im obigen Sinne bezeichnet einen Zustand, medienkompetent zu sein ist das Ergebnis eines Lernprozesses und besteht darin, über rein inhaltliches Wissen und ebenso rein inhaltliche Fähigkeiten zu verfügen. Die lebenslange Aufgabe der individuellen Persönlichkeitsentwicklung in der medialen Lebenswelt (vgl. Spanhel 2010, 51) ist aber nicht durch ein zu einem bestimmten Zeitpunkt aufgebautes Repertoire an Verfügungswissen zu bewältigen. Also greift Medienkompetenz als Zielwert der Medienpädagogik zu kurz, da sie alleine nicht hinreichend ist, den Prozess der medialen Selbstsozialisation in selbstbestimmter Weise zu durchlaufen. Die medienpädagogische Praxis, so die Kritik in einer Wendung zum Argument für Medienbildung weiter, muss dem Individuum also mehr anbieten als den Erwerb eines feststellbaren Medienkompetenzniveaus. Und dieses Mehr sollen Bildungsprozesse sein, in denen das Verfügungswissen der Medienkompetenz als Gegenstand einer kritischen Reflexion zugänglich ist, Orientierungswissen aufgebaut und immer wieder modifiziert werden kann. Aufgabe der Medienpädagogik muss also sein, Lernumgebungen zu schaffen, in denen Medienbildungsprozesse angestossen werden und stattfinden können (vgl. ebd., 53).

\section{Kritik des deskriptiven Arguments}

Als erste Prämisse des deskriptiven Arguments wird die Annahme getroffen, medienkompetent zu sein bestehe darin, rein inhaltliches Verfügungswissen aufgebaut zu haben. Mit Blick auf die in der erziehungswissenschaftlichen Debatte vorgeschlagenen Analysen des Begriffs der Medienkompetenz ist allerdings festzustellen, dass zahlreiche Charakterisierungen von Medienkompetenz wenigstens einen Aspekt beinhalten, der über im oben beschriebenen Sinne rein inhaltliches Verfü- 
gungswissen hinausgeht. So spricht z. B. Baacke von «Medienkritik» $(1996,120)$ als einem Bestandteil von Medienkompetenz, den er weiter in eine analytische, eine reflexive und eine ethische Unterdimension aufschlüsselt (vgl. ebd.). Auch in der Darstellung von Rosebrock und Zitzelsberger (2002, 150 ff.), die neben dem Ansatz Baackes fünf weitere zusammenfassen, sind durch alle Modelle hinweg solche Dimensionen enthalten, die das im Sinne der jeweiligen Analyse medienkompetente Individuum nicht nur zu den Inhalten des medialen Gegenstandsbereiches in ein Verhältnis setzen, sondern auch zu dem medialen Gegenstandsbereich selbst.

Vor dem Hintergrund dieser Beobachtung stellt sich die Frage, welche Formen von Orientierungswissen die Medienkompetenz als uneingelöste Desiderata offen lässt. Mit anderen Worten: Warum verfügt das im Sinne dieser Analysen medienkompetente Individuum lediglich über rein inhaltliches Wissen und ebensolche Fähigkeiten? Auf diese Frage wird später erneut einzugehen sein. Hier soll die Feststellung genügen, dass die fragliche Voraussetzung des deskriptiven Arguments dringender Klärung vor dem Hintergrund der Modellierungen von Medienkompetenz bedarf, die in der wissenschaftlichen Debatte diskutiert werden. Diesen liegt nämlich gerade kein «funktional-technologisches Kompetenzverständnis» (Tulodziecki 2011, 22) zugrunde, während eine solche Auffassung die Basis der zur Diskussion stehenden Prämisse zu sein scheint.

\section{Das normative Argument: Zweckorientierung vs. Selbstzweck}

Medienkompetent zu sein wurde oben beschrieben als Zustand, in dem das Individuum ein bestimmtes Mass medienbezogenen Verfügungswissens aufgebaut hat. An dieser Stelle ist eine Auffassung dieses Konzeptes naheliegend, unter der Medienkompetenz ein Bündel mehrerer Einzelkompetenzen ist (vgl. Schorb 2009, $52 \mathrm{ff}$.). Gestützt wird dieses Verständnis durch theoretische Arbeiten zur Medienkompetenz (vgl. z. B. Baacke 1996, 120; Rosebrock u. Zitzelsberger 2002, $150 \mathrm{ff}$. ), in denen der Begriff in Unterdimensionen aufgeschlüsselt wird, so dass der Eindruck entstehen mag, der empirische Zugang zur Medienkompetenz geschehe über einen wohldefinierten Katalog einzelner Bestandteile. Durch diese Analyse in Unterkompetenzen ist es möglich, den Erwerb von Medienkompetenz extern zu motivieren. Medienkompetenz ist nicht nur deshalb als erstrebenswertes Ziel anzusehen, weil sie der individuellen Persönlichkeitsentwicklung und einer reflektierten Selbstsozialisation dienlich ist - die sich aus dem deskriptiven Argument ergebenden strukturellen Bedenken seien hier vernachlässigt -, sondern ist darüber hinaus in vielen anderen Bereichen nützlich, z. B. für die Qualität der eigenen Leistungen im Studium oder die immer wichtiger werdende Profilierung auf dem Arbeitsmarkt. Wer kompetent im Internet nach Literatur suchen oder die Möglichkeiten sozialer Netzwerke ausnutzen kann, wer «Webdesign» oder «kompetenter Umgang mit Office-Anwendungen» in seinen Lebenslauf schreiben kann, scheint 
sich damit einen Vorteil gegenüber anderen erarbeitet zu haben. Medienkompetenz ist also nicht nur als normatives Ideal medienpädagogischen Handelns motiviert, sondern auch durch Zielwerte ausserhalb der Pädagogik.

Anders die Medienbildung: Sie ist gerade kein Zustand, wie oben diskutiert wurde, sondern ein prinzipiell offener Prozess, in dem sich das Individuum immer wieder von neuem selbst in seiner medialen Lebenswelt positioniert. Dabei halten sich Medienbildungsprozesse für sich selbst offen, indem sie das ständig im Aufbau befindliche Orientierungswissen als modifizierbar qualifizieren. Das Individuum soll in der Lage sein, «auch unter schwierigen Umständen frei zu denken und zu handeln» (Hugger 2006, 29), d. h., es soll in selbstbewusster Weise seine Persönlichkeit entwickeln und sein Verhältnis zu sich selbst und zur Welt bestimmen. Während medienkompetentes Handeln die Anwendung erlernter «Wahrnehmungs-, Gefühls-, Denk-, Handlung- und Wertungsmuster» (Spanhel 2010, 52) meint, werden diese Muster in Medienbildungsprozessen allererst ausgebildet (vgl. ebd.). Der einzige Zweck dieser Prozesse und damit die einzig infrage kommende Motivation für sie ist die Bildung der Persönlichkeit, d. h., Bildungsprozesse im Allgemeinen und insbesondere Medienbildungsprozesse sind Selbstzweck, für die es «kein von aussen 〈hinzufügbares〉 Bildungsziel» (Jörissen 2011, 221) gibt.

Während das oben rekonstruierte erste Argument auf der Diagnose einer strukturellen Unzulänglichkeit des Begriffs der Medienkompetenz in Bezug auf eines der übergeordneten Ziele pädagogischen Handelns beruht, liegt im Kern des nun formulierbaren zweiten Arguments ein normativer Anspruch gegenüber dem Zielwert wenigstens der Medienpädagogik. Vor allem in dem von Jörissen als «öffentlich-politisch-administrativ» $(2011$, 212) bezeichneten Diskurs birgt die Möglichkeit externer Motivation, die oben als Eigenschaft von Medienkompetenz diskutiert wurde, gleichzeitig die Gefahr externer Ziel- und Inhaltsvorgaben. Bei Übernahme von Medienkompetenz als Zielwert öffnet sich die Medienpädagogik in der Entwicklung und Bewertung ihrer Massnahmen dem Einfluss externer Interessen, z. B. zweckrationalen Anforderungen des Arbeitsmarktes (vgl. Hugger 2006, 31). Sie teilt dann die begriffliche Ebene mit solchen Akteuren, deren Ziel nicht die freie und selbstbestimmte Entwicklung der individuellen Persönlichkeit ist, deren Absichten diesem von der Pädagogik angestrebten Prozess schlimmstenfalls sogar entgegenwirken. Medienkompetenz ist daher als Zielwert der Medienpädagogik abzulehnen, da mit ihr ein Hauptanliegen der Pädagogik zugunsten einer Terminologie in den Hintergrund rücken würde, die an Kriterien der Zweckrationalität orientiert und somit pädagogisch inadäquat ist.

Die unausgesprochene normative Prämisse in dieser Argumentation scheint folgende zu sein: Zielwerte der Pädagogik, wenigstens in der Medienpädagogik, dürfen sich nicht zweckrationalistisch begründen lassen. Darüber wird später noch zu sprechen sein. Die Wendung dieser Kritik zum Argument für Medienbildung 
als Zielwert der Medienpädagogik ist offensichtlich: Medienbildung ist reiner Selbstzweck und damit immun gegenüber externen, d. h. ausserhalb der Pädagogik formulierten Anforderungen. Bildungsprozesse und somit insbesondere Medienbildungsprozesse sind weder funktionalisierbar noch planbar (vgl. ebd.). Aufgabe der Medienpädagogik ist es, wie oben bereits angesprochen, Lernumgebungen zu schaffen, die das Stattfinden medialer Bildungsprozesse begünstigen. Aber diese Prozesse lassen sich nicht gezielt initiieren oder auch nur vorhersagen (vgl. Jörissen 2011, 219). Sie sind lediglich ex post facto feststellbar und belegen dann die Qualität der medienpädagogischen Massnahme, die sie ermöglicht hat. Da sich Medienbildung auf diese Art den konkreten externen Anforderungen vor allem des oben genannten öffentlich-politisch-administrativen Diskurses entzieht, die immer planbar sein müssen und auf evaluierbare Ergebnisse zielen (vgl. ebd., $213 \mathrm{ff}$.), ist sie als Zielwert der Medienpädagogik nur rein pädagogisch und somit insbesondere nicht zweckrationalistisch zu begründen.

\section{Kritik des normativen Arguments}

Angenommen, die Bestimmung ihres Zielwertes als Medienkompetenz bringt die medienpädagogische Praxis tatsächlich auf eine gemeinsame begriffliche Ebene mit externen Akteuren, deren Interessen nicht die der Pädagogik sind. Und es sei ausserdem angenommen, dass als Konsequenz hieraus eine Einflussnahme dieser externen Akteure auf die Gestaltung und Bewertung medienpädagogischer Massnahmen möglich ist. Die Konklusion, Medienkompetenz sei als Zielwert abzulehnen, da sie zweckrationale Kategorien in die Medienpädagogik importiere, folgt unter diesen Prämissen aber vermutlich nur dann, wenn Zielwerte der Medienpädagogik externen Akteuren prinzipiell nicht zugänglich und d. h. mit zweckrationalen Kategorien prinzipiell nicht erfassbar sein dürfen. Die Qualität des normativen Arguments steht und fällt also mit dieser Zusatzannahme.

Die Begründung dieser Zusatzannahme scheint nun aber nur auf einem Weg erfolgen zu können: Zu zeigen ist eine Unverträglichkeit solcher Ziele, die zweckrational zugänglich sind, mit einem Hauptanliegen der Medienpädagogik, nämlich den selbstbestimmten Prozess der medialen Sozialisation zu begleiten. Ein Gegenbeispiel hierfür liefert jedoch die von Baacke (1996) vorgeschlagene Analyse von Medienkompetenz. In den Hauptdimensionen der Medienkunde, der Mediennutzung und der Mediengestaltung sind Unterdimensionen enthalten, deren jeweilige Ausprägung operationalisierbar und somit auch individuell evaluierbar ist, z. B. die «instrumentell-qualifikatorische Dimension» (ebd., 120, Hervorh. im Original) oder die «Programm-Nutzungskompetenz» (ebd.). Medienkompetenz im Sinne Baackes lässt sich zumindest teilweise auch in zweckrationalen Kategorien begründen. Bedienkompetenz, also die von Baacke als instrumentell-qualifikatorisch bezeichnete Unterdimension, lässt sich auch ausserhalb der Pädagogik als erstrebens- 
wert motivieren. Warum der Erwerb von Medienkompetenz in diesem Sinne dem genannten Hauptanliegen der Medienpädagogik im Wege stehen sollte, bleibt unklar. Für eine selbstbestimmte Positionierung des Individuums in der medial durchdrungenen Welt sind schliesslich auch Unterkompetenzen notwendig, die sich an den strukturellen Forderungen ebendieser Medienwelt orientieren. Schon dadurch lassen sie sich als zweckrational charakterisieren, doch dienen sie letztlich genau der Annäherung an das übergeordnete pädagogische Ideal. Vergleichbar ist diese Problemlage mit dem Verhältnis zwischen Lese- oder Schreibkompetenz und einem allgemeinen Bildungsideal im Sinne Humboldts: Letzteres ist nicht erschöpfend durch derartige operationalisierbare und sicherlich auch von ausserhalb der Pädagogik motivierbare Kompetenzen einzufangen. Doch auch wenn der Erwerb dieser Kompetenzen nicht im strikten Sinne notwendig für das Erreichen des übergeordneten Ziels sein mag, so ist er ihm doch in hohem Masse zuträglich. Bei genauerer Betrachtung erweist sich also wenigstens eine implizite Zusatzannahme, die den Schluss auf die gewünschte Konklusion erlauben würde, als bestenfalls fragwürdig und näherer Begründung bedürftig, schlimmstenfalls sogar als offensichtlich widerlegbar. Ist aber die hier diskutierte Prämisse nicht haltbar, muss die Lücke des normativen Arguments mit einer anderen Voraussetzung geschlossen werden.

\section{Drei Adäquatheitskriterien medienpädagogischer Zielwerte}

Sind obige Erwiderungen auf die vorgestellten Argumente erfolgreich, so ist wenigstens eine negative Verteidigung von Medienkompetenz als Zielwert der medienpädagogischen Praxis geleistet. Im Folgenden werden drei Adäquatheitskriterien für Zielwerte der medienpädagogischen Praxis vorgeschlagen, die als Bewertungsmassstab die Grundlage einer positiven Verteidigung von Medienkompetenz bilden können. Zuvor sei nochmals auf die systematische Grundannahme dieser Ausführungen verwiesen: Ein Hauptanliegen der pädagogischen Praxis im Allgemeinen ist es, die Entwicklung der individuellen Persönlichkeit und den Prozess der Sozialisation zu begleiten. Für die Medienpädagogik lässt sich damit eine Spezialisierung dieser allgemeinen Aufgabe ableiten, die zugleich das erste Adäquatheitskriterium liefert:

(1) Medienpädagogische Zielwerte müssen dem Hauptanliegen der Medianpädagogik zuträglich sein, das darin besteht, Prozesse der individuellen Persönlichkeitsentwicklung und der Sozialisation immer dort zu begleiten, wo sie im medialen Rahmen stattfinden.

Kriterium (1) ist von zentraler Wichtigkeit für jeden Zielwert, bleibt dabei aber doch nur eine Minimalanforderung. Eingangs wurde aus dem angenommenen Hauptanliegen der Pädagogik die Forderung nach einer theoretisch fundierten und praktisch anschlussfähigen Medienpädagogik begründet. Die Forderung nach 
theoretischer Grundlegung und praktischer Relevanz überträgt sich auch auf den Zielwert medienpädagogischer Praxis, so dass sich in Ergänzung zu (1) die folgenden beiden Adäquatheitskriterien ergeben:

(2) Medienpädagogische Zielwerte müssen theoretisch begründet sein, d. h. eine begriffliche Grundlage im Rahmen der Pädagogik haben.

(3) Medienpädagogische Zielwerte müssen praktisch anschlussfähig sein, d. h., sie müssen begrifflich eindeutige Ziele für die Praxis bestimmen, die

i. umsetzbar sind und

ii. Leitfäden für die Entwicklung und Bewertung medienpädagogischer Massnahmen formulieren.

Abschliessend werden nun die Konzepte der Medienbildung und der Medienkompetenz vor dem Hintergrund dieser Kriterien diskutiert.

\section{Medienbildung}

Im Hinblick auf Kriterium (1) scheint die Behauptung unstrittig, Medienbildung sei dem genannten Hauptanliegen der Medienpädagogik zuträglich. Dieses ist schliesslich, die im medialen Rahmen stattfindenden Prozesse der individuellen Persönlichkeitsentwicklung und der Sozialisation zu begleiten. Doch hier zeigt sich bei genauerer Betrachtung eine kategoriale Unklarheit: Wie kann die Medienpädagogik Medienbildungsprozesse begleiten, wenn diese doch der Zielwert sind, an dem die Entwicklung und Bewertung medienpädagogischer Massnahmen ausgerichtet ist? In Ergänzung zu Spanhel $(2010,53)$ liesse sich sagen, die Aufgabe der Medienpädagogik bestehe darin, Lernumgebungen zu schaffen, in denen Medienbildungsprozesse angestossen werden und stattfinden können, und diese Prozesse auch zu begleiten. Doch stellt sich dann die Frage, an welchem Zielwert die Begleitung dieser Prozesse ausgerichtet sein soll. Möglicherweise handelt es sich bei dieser Kritik am Konzept der Medienbildung als Zielwert der Medienpädagogik lediglich um eine terminologische Spitzfindigkeit, die sich leicht beheben lässt. Diese Frage soll daher hier nicht weiter vertieft werden, aber sie verdient eine Antwort.

Auch die theoretische Fundierung und damit die Adäquatheit von Medienbildung im Sinne von Kriterium (2) scheint prima facie offensichtlich zu sein, handelt es sich wenigstens bei dem enthaltenen Bildungsbegriff um eines der zentralen pädagogischen Konzepte. Auffällig ist hierbei zunächst nur eine hartnäckige Unschärfe dieses zentralen Begriffs (vgl. Hugger 2006, 30), die an das Konzept der Medienbildung vererbt zu werden scheint. Naheliegend ist in jedem Fall, die Haltbarkeit des allgemeinen Bildungsbegriffs als notwendige Voraussetzung an die des Medienbildungsbegriffs zu knüpfen, wobei eine Untersuchung des allgemeinen Begriffs den Rahmen dieser Ausführungen sprengen würde und daher nicht erfolgt. Festzustellen ist allerdings, dass auch der enthaltene Medienbegriff in verschie- 
denen Bedeutungen verwendet wird. Einerseits sind damit Medien in demselben Sinne gemeint, in dem sich auch die Medienkompetenz als medienbezogen versteht. Laut Tulodziecki ist der Medienbildungsbegriff dann aber «eher aus pragmatischen, denn aus bildungstheoretischen Gründen» $(2011,27)$ motiviert, so dass zu prüfen wäre, ob das so verstandene Konzept der Medienbildung Kriterium (2) erfüllt. Andererseits wird etwa von Spanhel der Medienbegriff derart liberalisiert, dass es ihm möglich ist, Medienbildung als den «Prozess und das Ergebnis der Reflexion der Medialität aller Bildungsinhalte und Bildungsprozesse» $(2010,53)$ zu bestimmen. Abgesehen von einer erneuten kategorialen Unschärfe - handelt es sich um einen Prozess oder um das Ergebnis eines Prozesses? - ist nun zu fragen, ob es sich hierbei noch um einen Zielwert, d. h. ein normativ gehaltvolles Ideal für die medienpädagogische Praxis handeln kann, ob also dieses Verständnis von Medienbildung in Einklang mit Kriterium (3) zu bringen ist. Ähnliches scheint für das Konzept der strukturalen Medienbildung (vgl. z. B. Marotzki u. Jörissen 2010; Jörissen 2011) zu gelten, das als rein deskriptiver Begriff zunächst normativ neutral bleibt.

In Bezug auf die praktische Anschlussfähigkeit des Begriffs der Medienbildung im Sinne von Kriterium (3) steht zu befürchten, dass die dem Bildungsbegriff eigene Unschärfe sowie die Unmöglichkeit, Bildungsprozesse gezielt zu initiieren oder vorherzusagen die Implementierung medienpädagogischer Massnahmen, die auf diesen Zielwert hin entwickelt sind, in erheblichem Masse erschwert (vgl. Hugger 2006, 30). Ausserdem sind Medienbildungsprozesse, d. h. nach den bisherigen Ausführungen Prozesse individueller Persönlichkeitsentwicklung und der Sozialisation, die im medialen Rahmen stattfinden und die Orientierungswissen in Bezug auf die mediale Lebenswelt erzeugen, zunächst normativ neutral und damit, wie oben bereits gesagt, nicht direkt als Zielwerte mit Kriterium (3) zu versöhnen. Das Konzept muss normativ angereichert werden, damit in der medienpädagogischen Praxis erkennbar ist, welche der formalen Entwicklungs- und Sozialisationsprozesse aus inhaltlichen Gründen anzustossen sind, denn sicherlich sind auch solche Prozesse denkbar, deren Tendenz z. B. in ethischer Hinsicht als problematisch einzustufen ist. In welcher Form diese Anreicherung erfolgen kann, wird hier nicht weiter diskutiert.

Festzuhalten sind gewisse Defizite, die das Konzept der Medienbildung als Zielwert der Medienpädagogik in Hinblick auf die oben formulierten Kriterien aufweist. Dabei soll nicht der Eindruck entstehen, es handele sich um prinzipielle und strukturelle Probleme, die nicht zu überwinden sind, doch es ergibt sich die Notwendigkeit, die begriffliche Grundlage und ihre Schnittstelle zur pädagogischen Praxis präziser zu fassen. Anderenfalls besteht die Gefahr, das medienpädagogische Handeln an einem unbeständigen und wenig belastbaren Ideal auszurichten. 


\section{Medienkompetenz}

Bezüglich der in Kriterium (1) ausgedrückten Forderung ist die folgende oben bereits getroffene Feststellung von zentraler Wichtigkeit: In einem pädagogisch reflektierten Diskurs über Medienkompetenz wird ihre Analyse gerade nicht auf ein rein inhaltliches Verfügungswissen verkürzt. Medienkompetenz im Sinne dieser Begriffsbestimmungen weist über reines Wissen-dass und Wissen-wie hinaus, indem sie immer auch eine reflexive Dimension enthält. Das bedeutet, der Erwerb zweckrational motivierbarer Unterkompetenzen ist nicht hinreichend für das Vorliegen von Medienkompetenz. Medienkompetent zu sein bedeutet nämlich auch, über Fähigkeiten zu verfügen, die «ebenso die kritische Aneignung von Medien [...] als auch eine mögliche Distanzierung» (Schorb 2009, 51) beinhalten. Mit anderen Worten: Durch die Förderung von Medienkompetenz lassen sich die Prozesse der individuellen Persönlichkeitsentwicklung und der Sozialisation begleiten, die im medialen Rahmen stattfinden.

Die theoretische Grundlegung von Medienkompetenz erfolgt bei Baacke vor den Hintergrund von Chomskys linguistischer Theorie einer universellen Grammatik und Habermas' Modell des idealen Diskurses. Diese beiden Ansätze werden, pädagogisch gewendet, im Ideal einer offenen und gleichberechtigten Kommunikation integriert, an der alle Individuen gemäss der ihnen eigenen kommunikativen Grundfähigkeit teilhaben können (vgl. Baacke 1996, 115). Die volle Ausbildung dieses Grundvermögens ist im Grenzwert die Medienkompetenz, die dem medienkompetenten Individuum die freie und selbstbestimmte Partizipation an seiner medialen Lebenswelt ermöglicht. Mit dieser begrifflichen Anbindung scheint das Desideratum theoretischer Fundiertheit im Sinne von Kriterium (2) erfüllt zu sein. Aber auch der Kompetenzbegriff im Allgemeinen, und damit die Medienkompetenz als ein Spezialfall davon, ist im theoretischen Diskurs der Pädagogik begründet (vgl. Tulodziecki 2011, 20 ff.), so dass es legitim zu sein scheint, in dieser Hinsicht eine positive Antwort auszusprechen.

Auch in Bezug auf Kriterium (3) scheint es keine Bedenken zu geben, Medienkompetenz als Zielwert zu empfehlen. Was als Ausgangspunkt des normativen Arguments beschrieben wurde, nämlich die Auffassung von Medienkompetenz als einem Bündel von zum Teil operationalisierbaren Unterkompetenzen, erweist sich hinsichtlich der Forderung nach praktischer Anschlussfähigkeit gerade als Stärke dieses Konzepts. So erlauben z. B. die oben genannten operationalisierbaren Unterdimensionen bei Baacke $(1996,120)$ die Formulierung konkret inhaltlicher Zielvorgaben unter dem allgemeinen Zielwert der Medienkompetenz. Medienpädagogische Massnahmen können z. B. mit dem Ziel entwickelt werden, die Bedienkompetenz oder das Wissen um organisatorische Zusammenhänge im medialen Raum auf ein bestimmtes Niveau zu bringen, und sie lassen sich in ihrer Qualität danach beurteilen, inwieweit sie diese Ziele erreichen. Notwendige Voraussetzung 
hierfür ist natürlich eine inhaltlich ausgestaltete Charakterisierung von Medienkompetenz, die im formalen Rahmen des Kompetenzbegriffs aber einfacher zu leisten ist, als vor dem Hintergrund eines Begriffs von Medienbildung, dessen prinzipielle Unbestimmtheit als eines seiner wesentlichen Merkmale gesehen wird (vgl. Hugger 2006, 30). Wie ebenfalls gesagt, ist der Erwerb dieser Kompetenzen nicht hinreichend für das Vorliegen von Medienkompetenz. Daraus ergeben sich weitere Konsequenzen für die medienpädagogische Praxis unter diesem Zielwert, die jedoch im positiven Sinne Leitlinien definieren und somit die Adäquatheit im Sinne von Kriterium (3) zusätzlich betonen: Um die Ausbildung der Kompetenzen zu fördern, die unter die reflexive Dimension fallen und nur schwer operationalisierbar sind, ist die Freisetzung intrinsischer Motivation erforderlich, was z. B. nach Baacke die Projektarbeit zum Mittel der Wahl in der Medienpädagogik macht (vgl. 1999, 91). Medienkompetenz bietet also nicht nur einen Katalog inhaltlicher und feststellbarer Unterziele, sondern formuliert gleichzeitig Anforderungen an die Methodik.

Zusammenfassend ist also festzuhalten, dass sich Medienkompetenz als ein Zielwert medienpädagogischer Praxis anbietet, der zumindest in Bezug auf die oben formulierten Kriterien tragfähig und fruchtbar ist. Wichtig ist hierbei natürlich eine entsprechend reichhaltige Charakterisierung dieses Konzepts, die aber auch der gemeinsame Nenner aller Analysen zu sein scheint, die im relevanten medienpädagogischen Diskurs vorgeschlagen werden.

\section{Fazit und Ausblick}

In den vorangegangenen Ausführungen zur Frage, ob Medienkompetenz oder Medienbildung der Zielwert medienpädagogischer Praxis sein sollte, wurden aus der in den Jahren 2009 und 2010 in der Zeitschrift merz: Medien + Erziehung geführten Debatte zunächst zwei Argumente gegen Medienkompetenz rekonstruiert, die zugleich für Medienbildung sprechen sollen. Das Ergebnis der kritischen Untersuchung dieser beiden Argumente lässt sich in spitzer Formulierung folgendermassen zusammenfassen: Die Skepsis gegenüber Medienkompetenz als Zielwert beruht auf einer begrifflichen Verwechslung. Das Verständnis von Medienkompetenz, das die öffentlichen, politischen und ökonomischen Diskurse bestimmt und an einem «anforderungsorientierten Qualifikationsbegriff» (Tulodziecki 2010, 50) orientiert ist, muss von dem Konzept unterschieden werden, das im Kontext der Medienpädagogik entwickelt und dort als Zielwert zur Diskussion gestellt wird. Letzteres lässt sich nicht nur gegenüber den vorgebrachten Einwänden verteidigen, sondern empfiehlt sich im Gegensatz zur Medienbildung sogar in positiver Weise als Zielwert medienpädagogischer Praxis. Die Basis für diese positive Verteidigung von Medienkompetenz waren der begriffliche Bezugsrahmen der oben genannten Debatte sowie drei Adäquatheitskriterien, die aus der Annahme abge- 
leitet wurden, eine vorrangige Aufgabe der pädagogischen Praxis sei es, Prozesse der individuellen Persönlichkeitsentwicklung und der Sozialisation zu begleiten. Weiterhin zu prüfen wäre somit, ob diese grundlegende Annahme über das allgemeine Ziel der Pädagogik, die hier ohne nähere Begründung getroffen wurde, haltbar ist. Bei einem positiven Ergebnis in dieser Frage, lassen sich auch die hier vorgeschlagenen Adäquatheitskriterien weiter schärfen und theoretisch festigen. Letztlich, so die Ansicht des Autors, sollten sowohl die Frage nach dem Zielwert der medienpädagogischen Praxis als auch die an anderer Stelle zu behandelnde Frage nach einem Leitbegriff medienpädagogischer Theoriebildung auf der Grundlage präzise formulierter und begrifflich belastbarer Kriterien entschieden werden.

\section{Literatur}

Baacke, Dieter. 1996. «Medienkompetenz - Begrifflichkeit und sozialer Wandel.» In Medienkompetenz als Schlüsselbegriff, hrsg. v. Ante von Rein, 112-124. Bad Heilbrunn: Julius Klinkhardt.

Baacke, Dieter. 1999. «Projekte als Formen der Medienarbeit.» In Handbuch Medien: Medienkompetenz - Modelle und Projekte, hrsg. v. Dieter Baacke, 86-93. Bonn: Bundeszentrale für Politische Bildung.

Fromme, Johannes. 2009. "Mediensozialisation/Medienbildung.» In Handbuch der Erziehungswissenschaft, Bd. III, hrsg. v. Hildegard Macha, Monika Witzke, Norbert Meder, Christina Allemann-Ghionda, Uwe Uhlendorff u. Gerhard Mertens, 931-938. Paderborn: Schöningh.

Fromme, Johannes und Benjamin Jörissen. 2010. «Medienbildung und Medienkompetenz - Berührungspunkte und Differenzen nicht ineinander überführbarer Konzepte.» merz: Medien + Erziehung 54 (5): 46-54.

Hugger, Kai-Uwe. 2006. «Medienkompetenz vs. Medienbildung? Anmerkungen zur Zielwertdiskussion in der Medienpädagogik.» In Methoden und Konzepte medienpädagogischer Projekte. Dieter Baacke Preis, Handbuch 1, hrsg. v. Jürgen Lauffer u. Renate Röllecke, 29-36. Bielefeld: GMK.

Jörissen, Benjamin. 2011. «〈Medienbildung〉 - Begriffsverständnisse und -reichweiten.» In Medienbildung und Medienkompetenz - Beiträge zu Schlüsselbegriffen der Medienpädagogik, hrsg. v. Heinz Moser, Petra Grell u. Horst Niesyto, 211-235. München: kopaed.

Marotzki, Winfried und Benjamin Jörissen. 2010. "Dimensionen strukturaler Medienbildung.» In Jahrbuch Medienpädagogik 8. Medienkompetenz und Web 2.0, hrsg. v. Bardo Herzig, Dorothee M. Meister, Heinz Moser u. Horst Niesyto, 19-39. Wiesbaden: VS Verlag für Sozialwissenschaften. 
Rosebrock, Cornelia und Olga Zitzelsberger. 2002. «Der Begriff Medienkompetenz als Zielperspektive im Diskurs der Pädagogik und Didaktik.» In Medienkompetenz - Voraussetzungen, Dimensionen, Funktionen, hrsg. v. Norbert Groeben u. Bettina Hurrelmann, 148-159. Weinheim: Juventa.

Schorb, Bernd. 2009. "Gebildet und kompetent. Medienbildung statt Medienkompetenz?» merz: Medien + Erziehung 53 (5): 50-56.

Spanhel, Dieter. 2010. «Medienbildung statt Medienkompetenz? Zum Beitrag von Bernd Schorb (merz 5/09).» merz: Medien + Erziehung 54 (1): 49-54.

Tulodziecki, Gerhard. 2010. «Medienkompetenz und/oder Medienbildung? Ein Diskussionsbeitrag.» merz: Medien + Erziehung 54 (3): 48-53.

Tulodziecki, Gerhard. 2011. «Zur Entstehung und Entwicklung zentraler Begriffe bei der pädagogischen Auseinandersetzung mit Medien.» In Medienbildung und Medienkompetenz - Beiträge zu Schlüsselbegriffen der Medienpädagogik, hrsg. v. Heinz Moser, Petra Grell u. Horst Niesyto, 11-39. München: kopaed. 